\title{
DISSIPATED ENERGY AS A FUNCTION OF MATERIAL MICROSTRUCTURE: TOWARDS OPTIMUM FRACTURE TOUGHNESS ${ }^{1}$
}

\author{
Mark J. Meisner \\ Institute for Mechanics and Materials \\ University of California, San Diego \\ 9500 Gilman Drive \\ La Jolla, California 92093-0404, USA \\ George N. Frantziskonis \\ Department of Civil Engineering and Engineering Mechanics \\ University of Arizona \\ Tucson, AZ 85721, USA
}

\begin{abstract}
The paper addresses material properties related to fracture-toughness, the focus being on brittle disordered materials. Through numerical lattice discretization based simulations of the rupture process the spatial variation of dissipated energy due to fracture is evaluated. Under certain conditions, its distribution is characterized by a multifractal spectrum $f(\alpha)$. As it happens, the multifractal spectrum, $f(\alpha)$, depends not only on the initial disorder present in the material, but also on the nature of the externally applied load. It is explicitly demonstrated that there is a direct connection between the multifractal spectrum of the dissipated energy and the total dissipated energy, due to mirco-crack formation during the rupture process. In other words, when the disorder of the material increases, a relevant parameter decreases, and the relative total dissipated energy decreases. The converse situation is also true. Finally, it is stressed that this work is amenable to experimental and further numerical verifications -- some paths toward this goal are discussed.
\end{abstract}

\section{Introduction}

Materials of technological or scientific interest are infrequently homogeneous. In many cases, the existence of spatial disorder is highly desirable -- e.g. for materials like concrete, rocks, ceramics, composites, and other brittle heterogeneous ones. Disorder may be due to the presence of different constituents such as aggregates, pores, micro-cracks, and interfaces. In other words, localized spatial variations in material properties are directly attributed to the material microstructure. A nonhomogeneous microstructure will have a significant effect on the fracture behavior of brittle materials; this is a subject of substantial technological importance.

The actual spatial disorders of the initial elastic and failure values are considered to form spatially correlated or uncorrelated random fields. Information on the statistical properties of such fields can be obtained: experimentally, through nondestructive evaluation methods (Dai

\footnotetext{
${ }^{1}$ Also includes an overview of parts of recent work by the authors.
} 
and Frantziskonis, 1994); techniques involving a "pure" geometrical basis (Breysse, et al., 1994) or provisionally (Schlangen and Van Mier, 1992; Murat, et al., 1992; Meisner, 1995; Meisner and Frantziskonis, 1995). As a result, knowing the (statistical) spatial disorders of the material allows use of a statistical mechanics approach to simplify and solve nonhomogeneous structures; in addition, materials subjected to intense external loading conditions may be tractable through the method presented in this paper.

The numerical process followed in this study is similar to the one found in the works of Herrmann, et al. (1989) and Hansen, et al. (1989) where two-dimensional beam, central force lattices were utilized, respectively, within the context of statistical mechanics, to study rupture of disordered media. In those studies a quenched spatially uncorrelated disorder in the breaking thresholds of the beams, bars, respectively, was considered. For both lattice configurations, the distribution of local forces just before the final completion of the rupture process, was found to be multifractal. At that instant, the moments, $M_{n}$, of order $n$ of the local forces $F$ can be characterized by a scaling exponent $y(n)$, that is $M_{n} \propto L^{y(n)}$ where $L$ is the size of the lattice. Consequently, it can be shown that the energy stored in the system, proportional to $F^{2}$ since the system is linear and elastic, is multifractal. This then implies that the complementary energy -the energy dissipated in the process of fracture -- should be multifractal. The dissipated energy is a measure on the fracture network, and this provides further physical interpretation of the multifractal properties -- provided the underlying fracture network is fractal -- the dissipated energy (measure) on it could possibly be multifractal (Mandelbrot, 1983). Since this energy is characteristic of the material fracture-toughness, it is interesting to study the distribution of dissipated energy. Frantziskonis (1994a; 1994b) studies this problem analytically in two dimensions. The analytical solutions suggest that indeed the dissipated energy can be multifractal. In particular, the so-called $D$-model multifractal was investigated. Since analytical solutions have only been obtained for certain cases of rather limited generality, in this study more general numerical results are presented, which extend those presented in Meisner (1995) and Meisner \& Frantziskonis (1995), where also the details of the numerical process followed can be found, thus it is not repeated herein.

The fracture process of brittle heterogeneous materials is investigated through the use of two-dimensional central force lattice model. In Section 2, a discussion of the lattice framework model with respect to the present study is presented. This is necessary since "traditional" fracture-toughness properties are defined within the context of elasticity (or continuum mechanics in a more general framework) thus a connection to the present study should be established. Also in Section 2, the concept of scaling is applied to find fracture surface/network related parameters. Next, the multifractal nature of the dissipated energy is illustrated in Section 3 . Finally, the demonstration of the correlation between the multifractal spectrum of the dissipated energy is performed in Section 4, which is followed by conclusions.

\section{The Numerical Model}

There are several methods to obtain numerical solutions of problems studied herein (a review of which is outside the scope of this paper). In the following, the lattice based numerical analysis used herein is discussed with respect to classical elasticity theory. This is so that the continuum mechanics based definitions and available results on toughness are compared to the present study. Furthermore, the continuum mechanics based on the analytical solutions 
presented in Frantziskonis (1994a; 1994b) can be compared to the results of the present numerical study. An appropriate lattice discretization, that is replacing the body of a material with a framework of bars (elements), is a consistent method for solution of elasticity problems, c.f. Hrennikoff (1941) as well as recent publications referred in the sequence.

The spatially dependent material properties are fixed before the loading (rupture) program begins; this is known as a quenched disorder process. Some of the local material characteristics which may be implemented into the lattice model are: density, elastic modulus, and failure strength. It has been shown through nondestructive experimental evaluation (Dai and Frantziskonis, 1994) that some heterogeneous brittle materials have a quasi-constant local density to local elastic modulus ratio. It is concluded that for length scales larger than a typical scale (that is, the grain size in a granular or polycrystalline material) it is rather the failure strength that varies spatially in a fashion important for fracture. Although further research in this important area is necessary -- cf. Dai and Frantziskonis (1994) for an extensive discussion -based on current knowledge and in order to reduce the number of possible material parameters, this condition will be assumed.

Each element is endowed with its own particular value of failure strength, $S$. Under the assumption that the elements are ideally linearly elastic and brittle, a bar will break when the force to which it is subject exceeds $S$ (for compression) or $T$ (for tension). The relation between the absolute values of the compression and tension parameters is given by $T=R S$, where $R$ may be defined for each element. However, for simplicity, only the case of symmetric rupture criterion is considered $(R=l)$. For simulation purposes, $S$ is considered, in general, to be a random field. The choice to consider spatial correlations in $S$ (a special case calls for $S$ being a spatially uncorrelated random variable) was based on many factors, which are explained in detail in Meisner and Frantziskonis (1995).

Despite the presence of very encouraging results in the literature, further research is needed to identify the statistical properties of actual materials precisely. In addition, the lack of complete information at this time should not, however, discourage investigations along the lines suggested herein. Thus, in the following $S$ is considered to be statistically stationary with a Gaussian autocorrelation function. Its expected value, variance, and (auto)correlation length provide, then, complete statistical information on $S$. In addition, the correlation length of $S, \ell_{s}$, is the radius of a circle beyond which $S$ ceases to be correlated (Yaglom, 1987).

The present approach to solid mechanics problem solving allows a natural induction of disorder into the model; and a physical interpretation of the algorithm is readily available. In other words, use of the lattice numerical model in conjunction with spatially correlated material properties suggest a connection between a purely statistical mechanics approach to fracture, that is as in Hansen et al. (1989), and a continuum/fracture mechanics approach which is based on homogenized values of material properties and crack propagation through a mathematically homogeneous solid. For infinitely long correlations the problem reduces to a continuum/fracture mechanics" one, while the spatially uncorrelated problem is a "pure statistical mechanics" one. Details of the solution process which can be found in Meisner (1995) and Meisner \& Frantziskonis (1995).

\subsection{Fracture Network Properties}

The concept of scaling has proven to be quite valuable in quantifying some of the properties of heterogeneous materials, thus the literature relevant to scaling properties of fracture 
networks/surfaces is very rich. Several experimental, numerical and theoretical studies have been reported, and for a recent review and trace of the literature we refer to Roux (1995). Despite the large number of publications on the subject, several issues are not well understood. For example, there is not, to our knowledge, a concrete physical explanation for the observed (experimentally) or calculated (numerically) fracture surface scaling properties. From this perspective, investigation of the associated dissipated energy as suggested herein is expected to provide further insight.

The fracture surface/network properties are defined through scaling exponents. In certain cases, there is a correlation between these exponents and toughness. Let us first briefly review the process of study followed herein, which is that of Matsushita and Ouchi (1989), and then discuss how it is "adapted" for particular cases. Suppose that a curve (i.e., a fracture network) is represented, in two-dimensional space, by $y=f(\alpha)$. Let $a(=1)$, be of unit length, defined as the smallest length scale of the curve. On the curve, two arbitrary points, $A$ and $B$, are chosen. The distance between $A$ and $B$ along the curve is measured and given in terms of $\mathrm{Na}$ $(=N)$. Next, the $x$ - and $y$-variances, $\operatorname{var}(x)$ and $\operatorname{var}(y)$, respectively, are calculated from measured points on the curve between $A$ and $B$. These variances are defined as:

$$
\begin{aligned}
& \operatorname{var}(x)=\frac{1}{N-1} \sum_{i=1}^{N}\left(x_{i}-\mu_{x}\right)^{2}, \\
& \operatorname{var}(y)=\frac{1}{N-i} \sum_{i=1}^{N}\left(y_{i}-\mu_{y}\right)^{2},
\end{aligned}
$$

where $\mu$ denotes mean or expected value and $\left(x_{i}, y_{i}\right)$ is the coordinate of the ith measured point on the curve. This procedure is repeated for many sets of points $A$ and $B$ on the curve. Finally, $\log$-log plots of the $x$ and $y$ standard deviations versus $N$ are used to see if they scale. Simulations of the fracture process are performed and ensemble averages of the quantities of interest are calculated. A complete report of the results can be found in Meisner (1995). In the following, dissipated energy, the central theme of this work, is examined.

\section{Multifractal Properties of the Dissipated Energy}

There are many physical quantities where in order to have an exhaustive characterization of their distribution, the implementation of an infinite series of scaling exponents may be necessary. Such generalized quantities can be equivalently described by the corresponding multifractal spectrum, that is in Schroeder (1991). In other words, a description of a distribution (measure) that is multifractal in nature may be described the corresponding $f(\alpha)$ versus $\alpha$ curve. Thus, the infinite set of scaling exponents are characterized by their singularities and the Lipshitz-Hölder exponent, $\alpha$, which determines the strength of the singularities. Consequently, a multifractal measure consists of interwoven sets of singularities which are characterized by their dimension that is given by $f(\alpha)$.

The case in which the crack dissipated energy of a brittle heterogeneous material is multifractal in nature is examined. The dissipated energy is quantified by employing the centralforce lattice model. Accordingly, the multifractal spectrum must be defined in terms associated 
with the lattice model in a rather "standard" fashion, that is, as presented by Charmet $e t$ al. (1990, Chapter 2).

As mentioned above, it is possible to describe the crack dissipated energy in terms of an infinite set of scaling exponents or by its Legendre transformation -- the $f(\alpha)$ spectrum. Accordingly, i.e. (Charmet, et al., 1990; Chapter 2) the study is made by plotting $\log \left(N_{i}(E, L)\right) / \log (L)$ versus $\log (E) / \log (L)$, the rescaled $\log$-histogram of the dissipated energy distribution. As a result, if the distribution is not multifractal, only a single point will be obtained; otherwise, the resulting curve will tend to be size-independent for large lattice sizes. It is made clear that the distribution of energy (energy released by each element that has been broken) at rupture is examined, that is, at the point where the multifractal properties of the load distribution are studied, for example, Hansen, et al. (1989). Furthermore, it is noted that for the case of extreme disorder, that is, when $\ell_{s} / L \rightarrow 0$ and the C.O.V. of the failure threshold is extreme, would be a percolation threshold and the dissipation energy will be multifractal (Roux and Hansen, 1989). For other cases, that is, for the ones studied in our work -- where $\ell_{s} / L$ has a nonzero value and the disorder is not extreme -- at rupture (where the dissipated energy is studied) we have a sort of "specialized" percolation case, in the sense that a connection of broken bars from one side of the specimen to the other is formed.

The method of examining the possibly multifractal properties of dissipated energy is similar to that reported by Hansen, et al. (1989) and Herrmann, et al. (1989). Figure 1 shows a typical rescaled log-histogram of the dissipated energy distribution at the failure state for different lattice sizes. A good collapse of the data, especially with increasing lattice size, is observed. It is noted that the number of simulations employed decreased with increasing lattice size. The numerical data associated with the highest of energies is not shown in this and in subsequent figures, due to finite size effects. In other words, the collapse of the data for high energies (small $\alpha$ ) is unacceptable. There are two possible reasons for this. First, the number of broken elements with very high energies is small compared to the total number of broken bars, and hence, a good statistical sample is not obtained. Second, localized clusters of high energy regions may be too large compared to the lattice size for appropriate stochastic analysis. uniaxial ransion

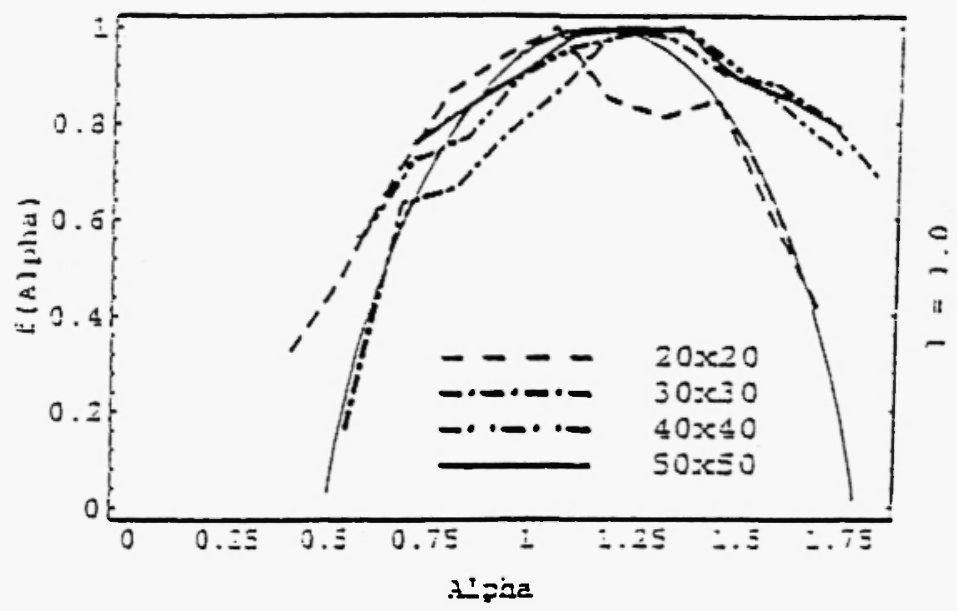

Figure 1: Rescaled log-histogram of the dissipated energy from four different lattices $(L=$ $20,30,40,50)$ subjected to uniaxial tension. The Coefficient of Variation of $S$ is 0.2 and $\ell_{s} / L=1$. The thin solid line corresponds to the so-called $D$-model multifractal, and is discussed further in the sequence. 
In Figure 1, a thin solid line corresponds to the $p$-model $f(\alpha)$ curve and it is discussed subsequently. Although the lattices considered are rather small to make any definite conclusions, the fact that these plots tend to be size-independent (for increasing lattice size, although the "collapse" is not shown herein for clarity in the figures) provides evidence that the dissipated energy distribution is multifractal. It is noted that the log-histogram collapsed into a single point (which implies a homogeneous fractal) for the zero disorder cases, where C.O.V. $=0$.

An analytical solution to the problem of the crack dissipated energy would be useful for comparison with the numerical approach of the lattice formalism, as well as the understanding of brittle fracture. Under certain conditions, there is a strong possibility that the dissipated energy distribution is multifractal (Frantziskonis, 1994a; 1994b). The proposed model is the (commonly used) so-called $p$-model (Meneveau and Sreenivasan, 1987). Using the notation of Meeneveau and Sreenivasan (1987) and Frantziskonis (1994b), the generalized dimensions are given by:

$$
D_{q}=\left\{p_{1}^{q}+\left(1-p_{1}\right)^{q}\right\}^{\frac{1}{1-q}}
$$

where $p_{1}$ designates normalized dissipated energy along a (straight) crack of length equal of the lower cutoff considered in the analysis. A value of $p_{1}=0.5$ indicates a homogeneous fractal, while the lower its value the higher the spatial disorder in the dissipated energy. Given the value of $p_{1}$, the expression for the multifractal spectrum is given in terms of a dimensionless parameter $\zeta$,

$$
\alpha=\frac{\log _{2}\left(p_{1}\right)+(\zeta-1) \log _{2}\left(p_{2}\right)}{\log _{2}\left(l_{1}\right)+(\zeta-1) \log _{2}\left(l_{2}\right)}
$$

and

$$
f(\alpha)=\frac{(\zeta-1) \log _{2}(\zeta-1)-\zeta \log 2(\zeta)}{\log _{2}\left(l_{1}\right)+(\zeta-1) \log _{2}\left(l_{2}\right)}
$$

where $\ell_{1}=\ell_{2}=1 / 2$ and $p_{2}=1-p_{1}$, for the $p$-model (Meneveau and Sreenivasan, 1987).

The parameter $\zeta$ may be eliminated, giving the explicit expression for $f(\alpha)$ as a function of $\alpha$, with only one variable, $p_{1}$. Under certain conditions it was possible to find the value of $p_{1}$ analytically (Frantziskonis, 1994b). The analytical solutions are approximate and were obtained under several simplifying assumptions. For compllete details on the connection between the analytical and numerical solutions we refer to Meisner \& Frantziskonis (1995).

In closing this section, we discuss some points relevant to the use of $f(\alpha)$ as a measure of toughness. Given the evidence of multifractal distribution of dissipated energy, the total energy dissipated in a specimen will be strongly dependent on its underlying distribution, which, in turn, is related to the initial heterogeneity of the material. Thus, the present approach shows promise in establishing a fundamental relation between material microstructure and toughness. As another implication, toughness is shown to be size/shape dependent as well as dependent on the external loading conditions. Another aspect where the properties of the dissipated energy 
distribution are of use is in identifying what parts -- that is, the "strong" or the "weak" ones in a gross sense -- of a material are the major toughness contributors. Experience, c.f. discussion in Dai and Frantziskonis (1994) has provided evidence that, depending on the specific spatial disorder present, some materials dissipate energy mainly in their "strong" regions, while others do so in their "weak" ones. We believe that work along the lines of the present paper provides a fundamental framework for studying such important tasks closely. Of course, the present work is amendable to several possible experimental verifications. A combination of non-destructive, destructive, and numerical work seems to be promising. Several possibilities for extension and/or verification of the present work exist, an example is given in the following. Nondestructive evaluation techniques can identify the initial spatial distribution of material properties. Then, a combination of (experimental) investigation of the fracture network/surfaces and numerical analysis of the problem of the distribution of dissipated energy can provide the relevant $f(\alpha)$. This should provide a relation among initial material heterogeneity -- properties of the fracture surface/network -- and energy dissipated for its development. By comparing with the total energy dissipated in a sample (measured experimentally) and by studying the cracks not interconnected with the main one(s), information on the so-called fracture process "zone" can be obtained. This is partly the reason that cracks not connected with the main one(s) were not included in the present analysis (of the dissipated energy distribution). It seems that for some materials, such a process zone is important, while for other ones it is negligible; and this needs to be investigated on a fundamental basis.

\section{Influence of Spatial Correlations and Loading Conditions on the Dissipated Energy}

As demonstrated in the previous sections, for both slow and fast crack propagation cases, there is a fracture network which will have fractal and multifractal characteristics. In this section, a connection between some of these characteristics and the total dissipated energy of the fractured lattice structure is made. This connection is only possible with explicit dependence on the statistical properties of the heterogeneity of the $S$ field before the rupture process begins and the external displacement loading conditions. In other words, simply, the dissipated energy is the result of stress relief, and this may lead to additional insight on the fracture toughness of heterogeneous materials. In addition, the dependence of the spatial correlations and loading conditions on the dissipated energy is studied in detail; in the following sequence, the results of this examination, of the characteristics of the total dissipated energy, is related to the multifractal $p$ model, through the parameter $p_{1}$.

Similarly, as before, simulations of the fracture process are performed, and ensemble averages of the quantities of interest are calculated. The mean of the $S$ fields have been defined to be unity, and the Coefficient of Variation (C.O.V.) is taken to be 0.2 for all the simulations use to obtain the results of this section. The values of the total dissipated energy are determine for three displacement loading cases -- uniaxial tension, dilatation, and shear. Consequently, the results of these simulations are presented in Table 2.

This table was generated from 20 numerical realizations, for each case, of $60 \times 60$ lattice size structures. Please see Figure 2 for an example of the lattice structure. Consequently, there are several major trends in the data that may be identified, for varying the ratio of the correlation length and the total lattice size -- that is, $\ell_{s} / L=1,1 / 2,1 / 15,1 / 30$. The fluctuations of the realizations were the same throughout all the simulations, C.O.V. $=0.2$. For $\ell_{s} / L=1$, 
$1 / 2$ the structures is considered to be relatively highly correlated. Conversely, for $\ell_{s} / L=1 / 15$, $1 / 30$ (where the numerical correlation length is small compared to the element (bar) size), the structure is considered to be relatively weakly correlated. In Figure 3(a) and 3(b), examples of the initial random $S$ fields are given. A strongly correlated field is illustrated in Figure 3(a); conversely, a weakly correlated structure is demonstrated in Figure 3(b).

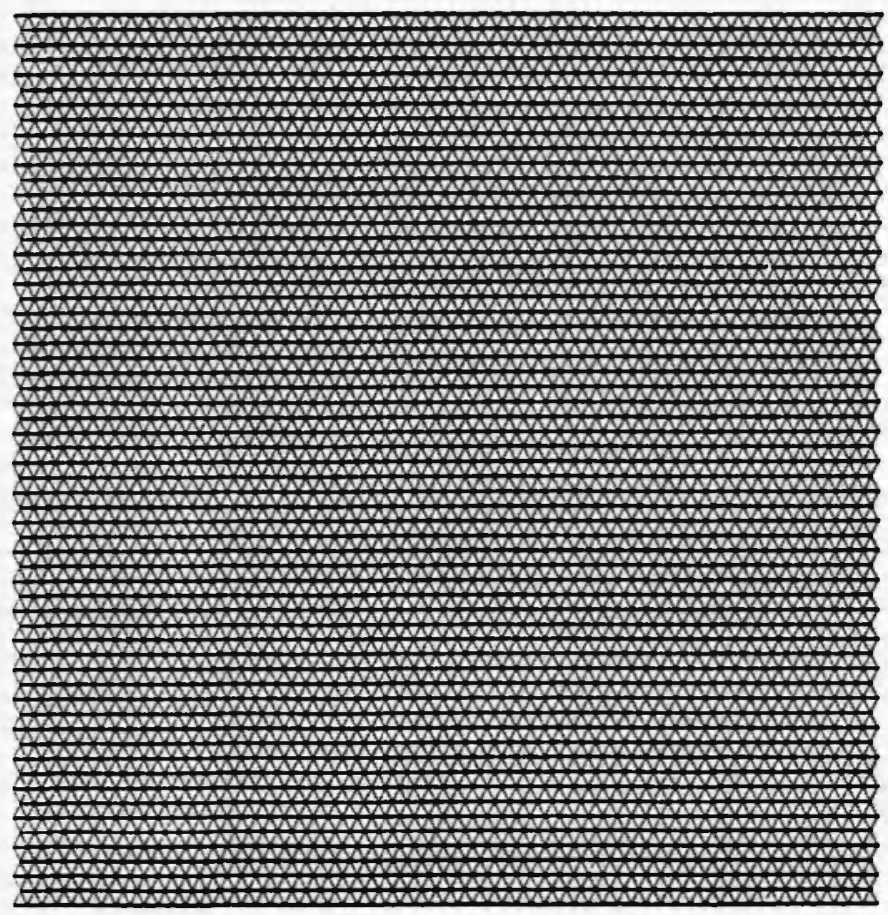

Figure 2: Example of the $60 \times 60$ lattice structure.

Table 2. Total Dissipated Energy $(60 \times 60$ lattice $)$, C.O.V. $=0.2$

\begin{tabular}{|c|c|c|c|c|}
\hline & Uniaxial Tension & Dilatation & Shear & \\
\hline$\ell_{s} / L=1$ & $2.9 \times 10^{7}$ & $4.1 \times 10^{8}$ & $1.3 \times 10^{7}$ & \multirow{2}{*}{$\begin{array}{l}\text { Relatively } \\
\text { Strongly } \\
\text { Correlated }\end{array}$} \\
\hline$\ell_{s} / L=1 / 2$ & $2.5 \times 10^{7}$ & $3.9 \times 10^{8}$ & $1.2 \times 10^{7}$ & \\
\hline$\ell_{s} / L=1 / i 5$ & $5.1 \times 10^{8}$ & $3.8 \times 10^{8}$ & $7.2 \times 10^{6}$ & \multirow{2}{*}{$\begin{array}{l}\text { Relatively } \\
\text { Weakly } \\
\text { Correlated }\end{array}$} \\
\hline$\ell_{s} / L=1 / 30$ & $2.9 \times 10^{8}$ & $2.2 \times 10^{8}$ & $1.8 \times 10^{6}$ & \\
\hline
\end{tabular}

The first major trend that is identified is as the relative correlation length, $\ell_{s} / L$, decreases the total dissipated energy of the fractured structure decreases for the dilatation and shear displacement loading cases. For the uniaxial case, the total dissipated energy is shown to be extremely dependent on the variation of $\ell_{s} / L$. Accordingly, the results are directly supported by the data presented in Meisner \& Frantziskonis (1995) An explicit demonstration of the effects of the displacement loading on the same heterogeneous structure is shown in Figures 4(a), 4(b), and 4(c) -- corresponding to uniaxial tension, dilatation and shear, respectively. 


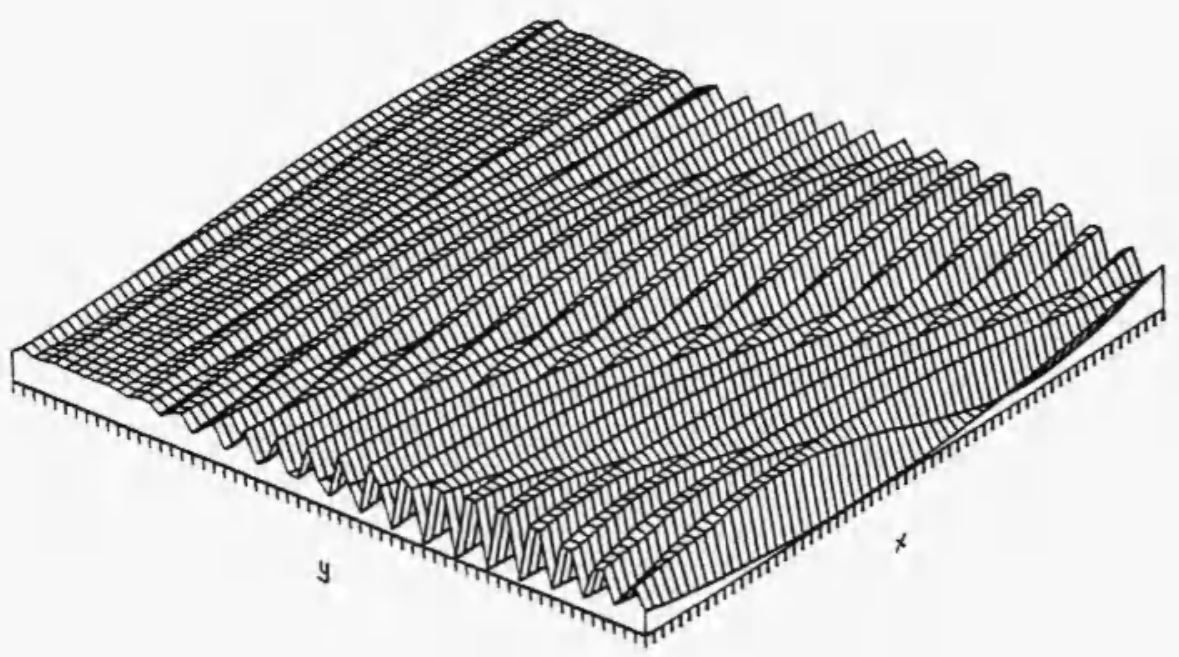

(a) $\ell_{5} / L=1$, strongly correlated

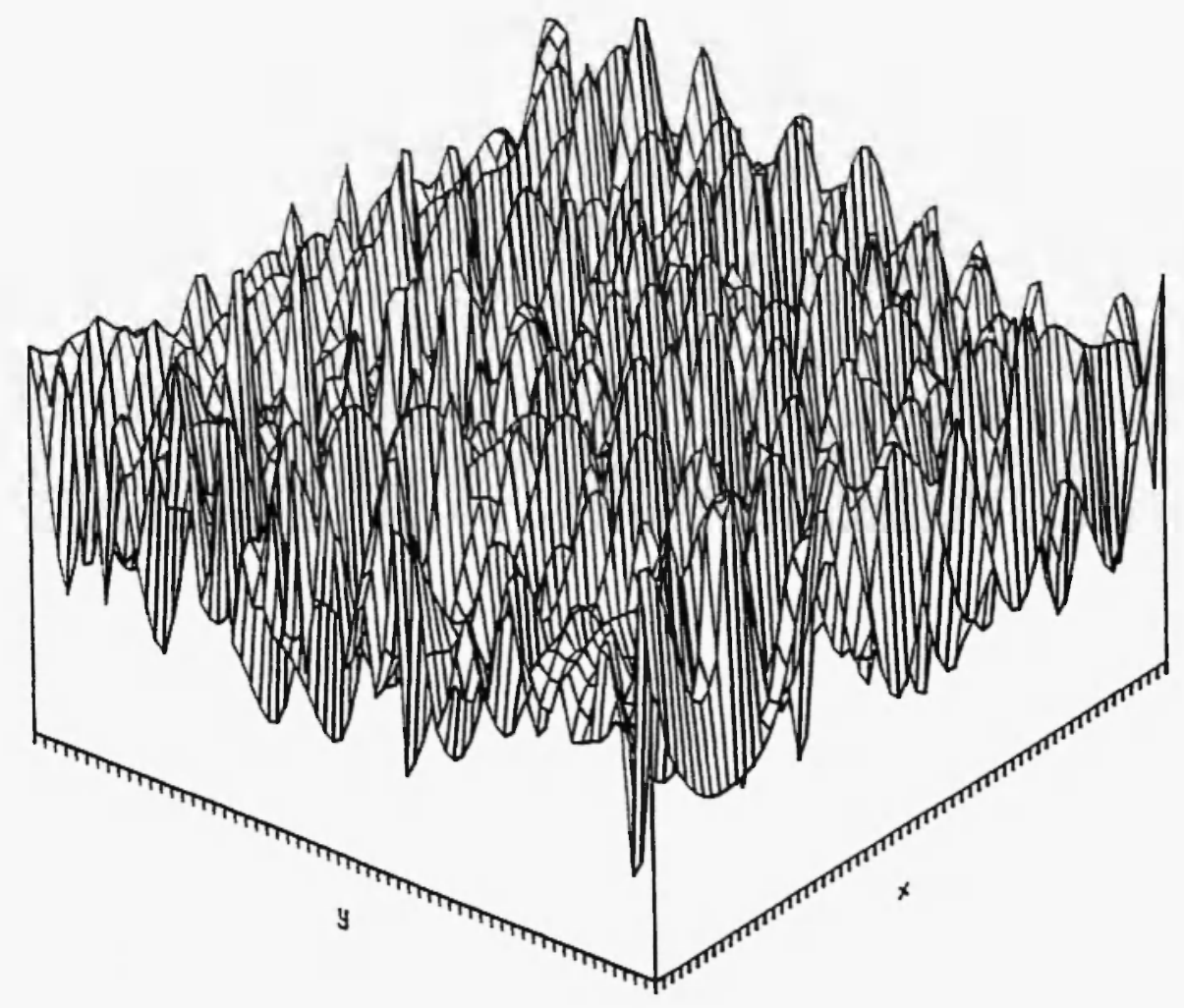

(b) $\ell_{s} / L=1 / 30$, weakly correlated.

Figure 3: Examples of the initial random $S$ field, C.O.V. $=0.2$ 


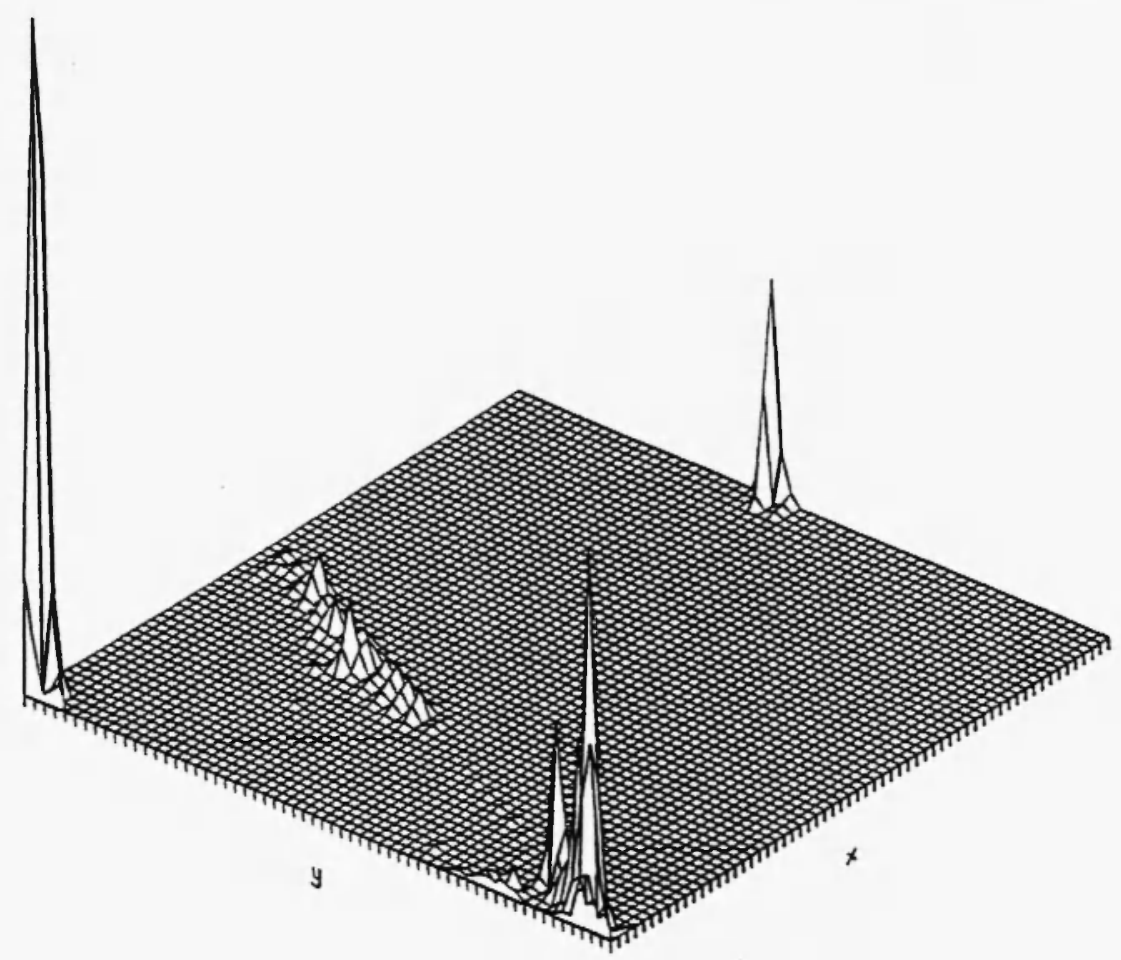

(a) uniaxial tension

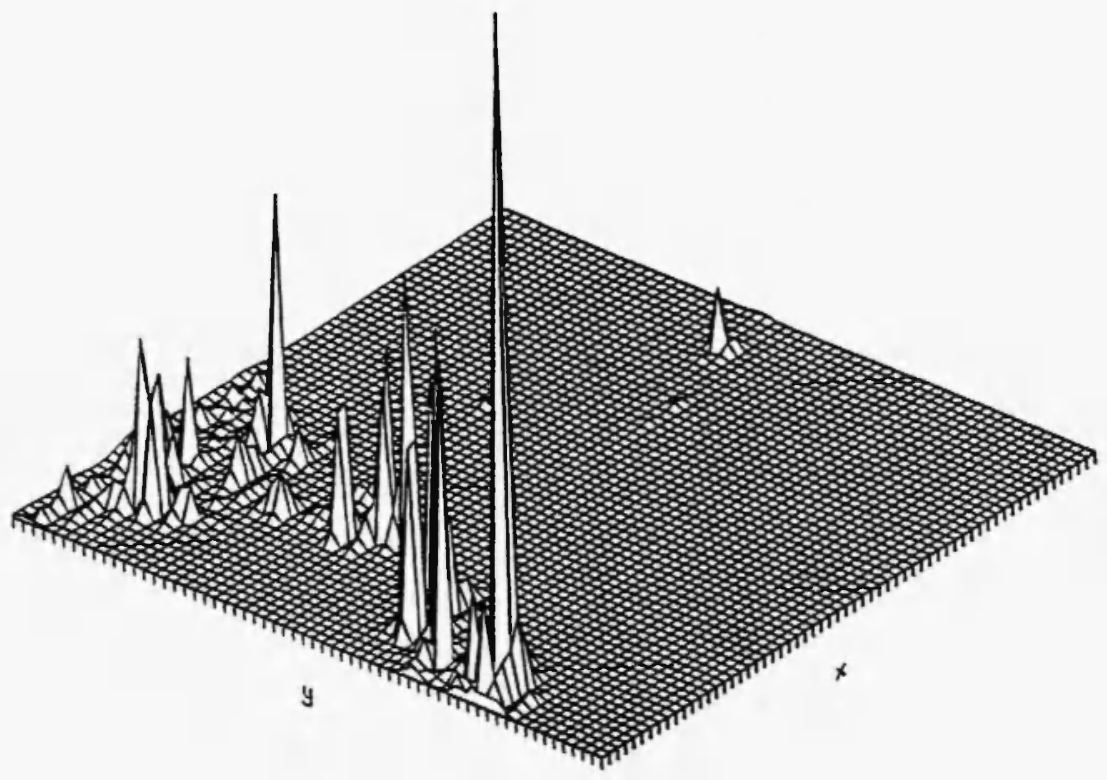

(b) dilatation

Figure 4: Dissipated energy of the ruptured elements of the lattice structure, C.O.V. $=0.2$ and $\ell_{s} / L=1$ 


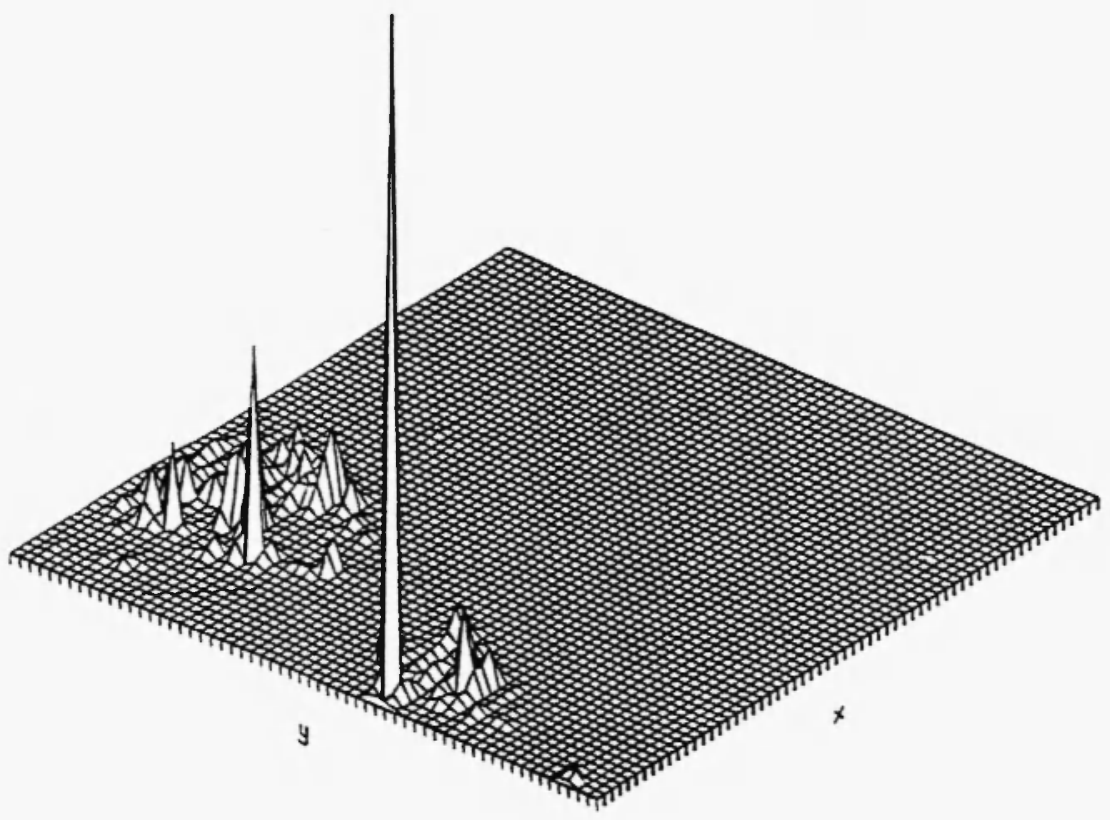

(c) shear

Figure 4 (cont'd): Dissipated energy of the ruptured elements of the lattice structure,

$$
\text { C.O.V. }=0.2 \text { and } \ell_{s} / L=1
$$

It has been shown in the previous section that the disorder of the system is directly related to the $p$ model parameter $p_{1}$. In other words, $p_{1}$ is an explicit function of the fluctuation, C.O.V., and the relative correlation length, $\ell_{5} / L$, of the $S$ fields (Frantziskonis, 1994b; Meisner and Frantziskonis, 1995). What is more, the lower $p_{1}$ value the higher the disorder exist of the system. From the data of Table 2, it may be concluded that for relatively strongly correlated structures the total dissipated energy decreases in the order of: dilatation, uniaxial tension, and shear displacement loading conditions. In addition, on the contrary, for weakly correlated material structures the total dissipated energy decreases in the order of: uniaxial tension, dilatation, and shear loading. To illustrate these findings Figures 5-7 -- which correspond to uniaxial tension, dilatation and shear respectively -- are presented. The relationship between disorder, $\ell_{s} / L$, and the loading conditions to the rupture profiles is now explicitly demonstrated, through these figures.

In short, a direct relationship between the parameter $p_{1}$, the disorder of the system, and the total dissipated energy has been developed. This relationships may be restated simply as: when the disorder of the material increases, the parameter $D_{1}$ decreases, and the relative total dissipated energy decreases. By the same token, when the disorder decreases the parameter $p_{1}$ will tend towards 0.5 and the total dissipated energy will also increase. This may be illustrated as:

disorder of the material increases(decreases)

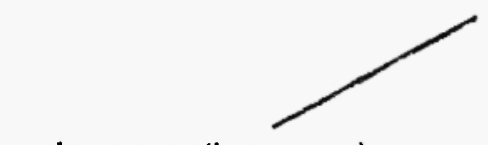

$p_{1}$ decreases(increases)

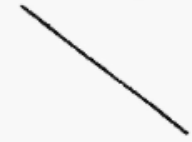

total dissipated energy decreases(increases) 


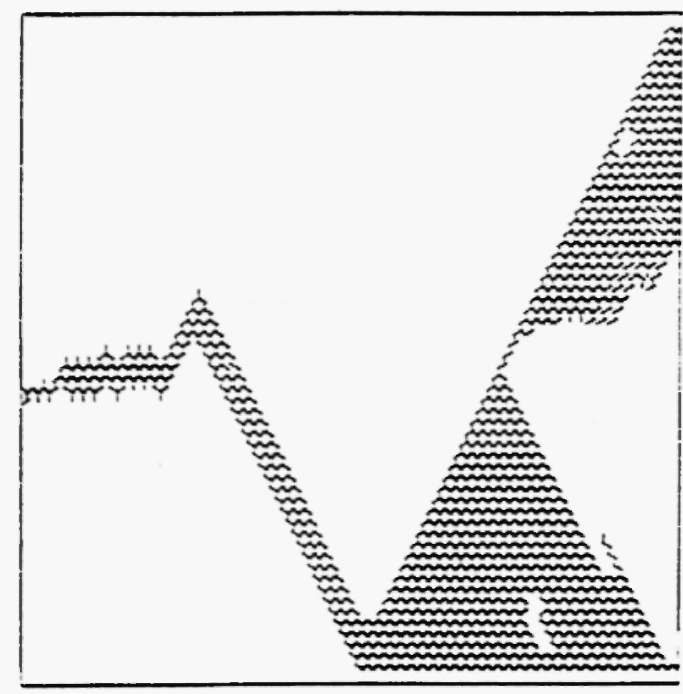

(a) $\ell_{s} / L=1$

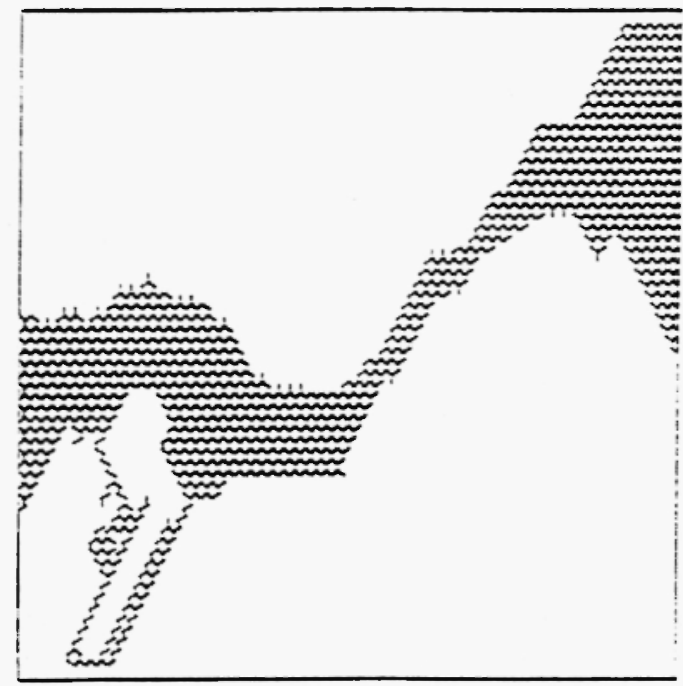

(b) $\ell_{s} / L=1 / 30$

Figure 5: Fractured heterogeneous lattice structure under uniaxial tension. C.O.V. $=0.2$ 


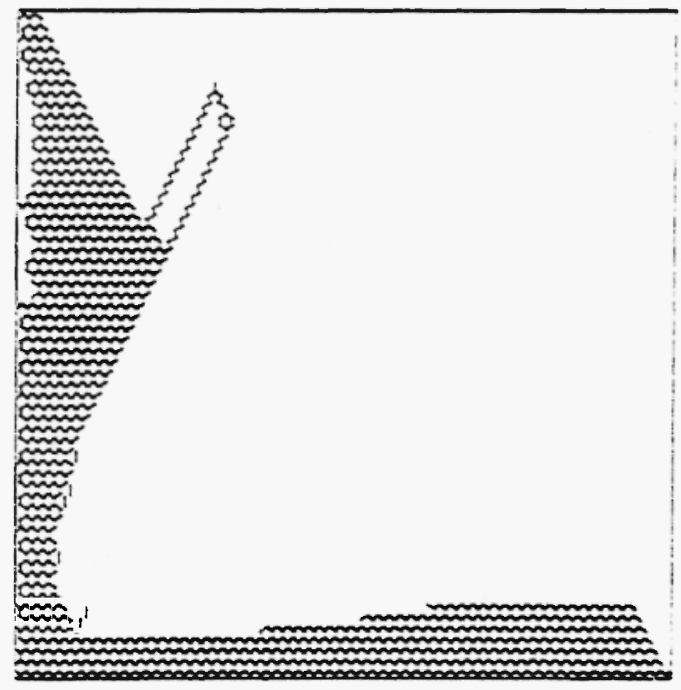

(a) $\ell_{5} / L=1$

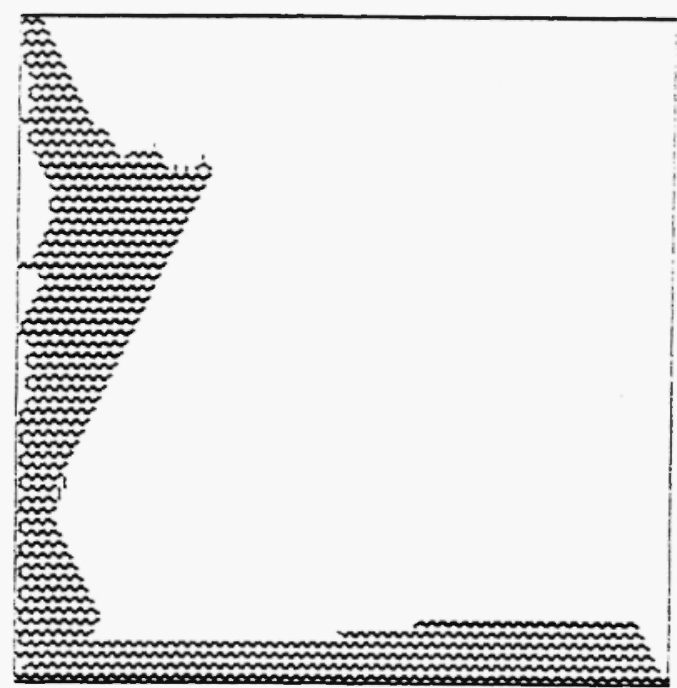

(b) $\ell_{s} / L=1 / 30$

Figure 6: Fractured heterogeneous lattice structure under dilatation. C.O.V. $=0.2$ 


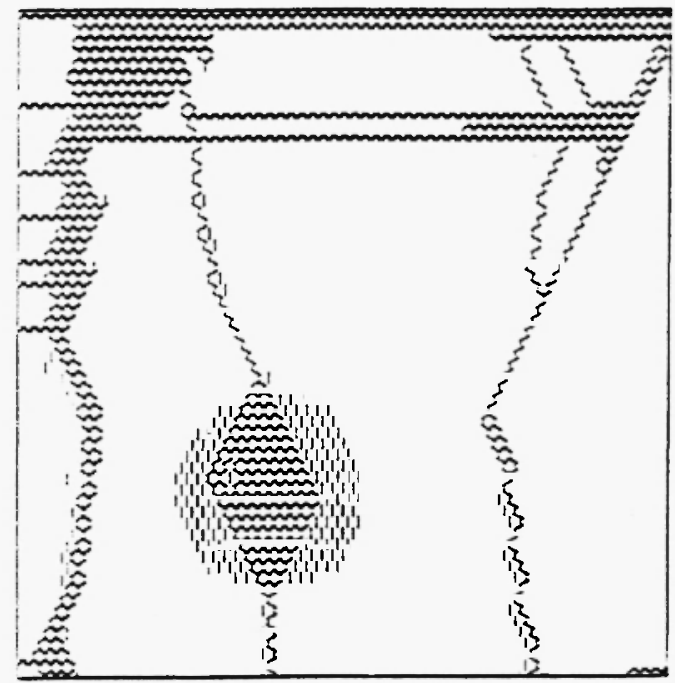

(a) $\ell_{s} / L=1$

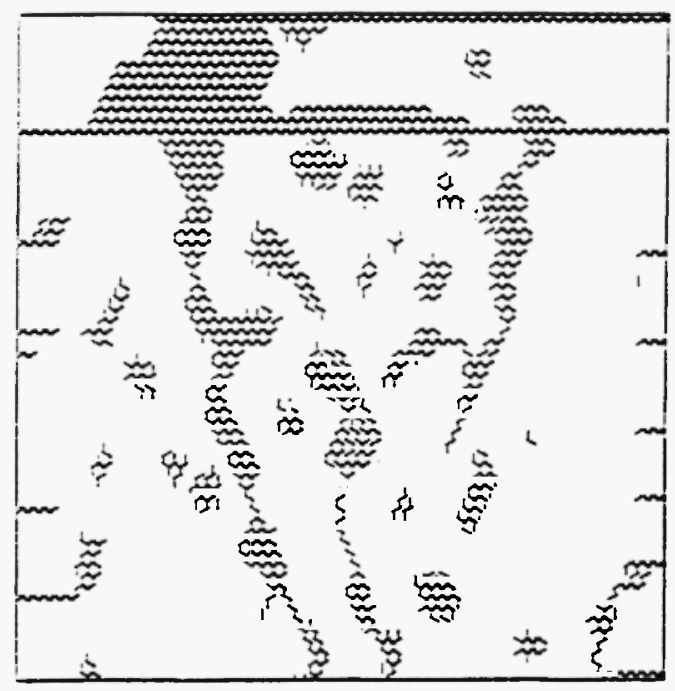

(b) $\ell_{s} / L=1 / 30$

Figure 7: Fractured heterogeneous lattice structure under shear. C.O.V. $=0.2$ 


\section{Conclusions}

Through a lattice based numerical approach, renewed fracture-toughness related parameters are suggested. In particular, the $f(\alpha)$ curve of the dissipated energy shows considerable promise in serving as a material, structure, as well as load path dependent set of characterization parameters. Thus, it is suggested herein that fracture-toughness should not be defined independently of the nature of the external load applied to a structure of even independently of its geometry (size, shape). Furthermore, the $p$-model, when applicable, provides an ever further simplified description in terms of one number, namely $p_{1}$. In passing, an important connection between a purely statistical mechanics and a continuum based approach to the problem of rupture and toughness is addressed.

\section{Acknowledgements}

The present research was supported partially by a grant from the Institute for Mechanics and Materials and the Japan Society for the Promotion of the Sciences, in conjunction with the National Science Foundation (NSF) to MJM; and by grants from NSF and the Hughes Aircraft company to GNF.

\section{References}

Breysse, D., Fokwa, D., and Drahy, F., 1994, Appl. Mech. Rev., 47, (p2) S184.

Charmet, J.C., Roux, S., and Guyon, E., Eds. 1990 Disorder and Fracture. Plenum Press, New York.

Dai, H., and Frantziskonis, G.N., 1994, Mech. Mater. 18, 103

Frantziskonis, G.N., 1994a, Eur. J. Mech. A/Solids 1373

Frantziskonis, G.N., 1994b, Appl. Mech. Rev., 47 p2 S132

Hansen, A., Roux, S., and Herrmann, H.S. 1989 J. de Physique 50733

Herrmann, H.J., Hansen, A., and Roux, S. 1989 Phys. Rev. B. 39637

Hrennikoff, A., 1941 J. App. Mech. 8 A169

Mandelbrot, B.B. 1983 The Fractal Geometry of Nature, Freeman, San Francisco

Matsushita, M. and Ouchi, S. 1989 Physica D. 38246

Meisner, M.J., 1995, Ph.D. Dissertation, University of Arizona

Meisner, M.J. and Frantziskonis, G.N. 1995 Multifractal fracture-toughness properties of brittle 
heterogeneous materials, preprint

Meneveau, C., and Sreenivasan, K.R., 1987 Phys. Rev. Lett., 591424

Murat, M.M., Anholt, M., and Wagner, H.D. 1992 J. Mater. Res. 73120

Roux, S. and Hansen, A. 1989 Europhys. Lett. 8729

Roux, S. 1995 Rugosites de Fracture: Observations et speculations Proc. MECAMAT Aussois, Fran, Janvier 95

Schroeder, M. 1991 Fractals, Chaos, Power Laws: Minutes from an infinite paradise, Freeman, New York

Yaglom, A.M., 1987, Correlation theory of stationary and related random functions SpringerVerlag, New York 